\title{
Intermediate-mass fragment production in spallation reactions
}

\author{
M. Valentina Ricciardi ${ }^{1}$, Aleksandra Kelić ${ }^{1, a}$, and Karl-Heinz Schmidt ${ }^{1}$ \\ GSI, Planckstr. 1, 64291 Darmstadt, Germany
}

\begin{abstract}
Two mechanisms for the production of intermediate-mass fragments (IMF) were implemented in the statistical-model code ABLA07. At low excitation energies IMFs are emitted as evaporated nuclei. If the excitation energy of the system exceeds a given threshold, IMFs are formed in the simultaneous break-up of the system, modelled according to a power-law distribution, which is suggested by several theoretical models. The improved code was benchmarked on a large amount of experimental data, among which the high-precision data measured at GSI.
\end{abstract}

\section{Introduction}

The precise knowledge of the production cross-sections of all nuclides produced in proton- or neutron-induced reactions up to the $\mathrm{GeV}$ regime is of extreme importance for the design of an accelerator-driven system, and for many other technical applications, like neutron sources, radioactive-ion beam facilities, and space technology. Also in astrophysics, these cross sections are important as they enter into the description of the processes that affect the composition of energetic nuclei during their transport through the Galaxy, from their source to the Earth where they are observed. Considering the large number of different target materials and energies at which these reactions can occur, it would be an enormous effort to provide all the required nuclear data from experiments. In this scenario, the best solution is to develop nuclear-reaction codes with high predictive power. To guarantee the reliability of the calculations for every target-projectile-energy combination computational tools based on parameterisations have to be avoided. Codes based on physical models are mandatory, although computing time represents often a limit. An optimum solution is represented by nuclear reaction codes based on the statistical model, which have high physics content but are still relatively fast.

During the last ten years a large body of experimental data concerning spallation residues was obtained in devoted experimental campaigns [1-5]. Parallel to the experimental campaign, nuclear-reaction codes were developed and widely benchmarked on the experimental data. In the frame of a European project [6], two codes working in the $100-2000 \mathrm{MeV}$ energy range were further improved: the statistical-model code ABLA (see ref. [7] and therein references), which describes the statistical de-excitation of a heated nucleus, and the intranuclear-cascade model INCL4 [8], which describes the first stage of the spallation reaction.

Here we report on the progress in the development of the ABLA code (current version: ABLA07) concerning the production of very light fragments. The range of emitted fragments was extended above $\mathrm{Z}=2$ in order to obtain a more realistic description of the production of intermediate-mass

${ }^{a}$ Presenting author, e-mail: email@address.zz fragments (IMFs), which was strongly underestimated in the previous version. Two mechanisms for the production of IMFs were implemented. The first concerns the evaporation of IMFs from a heavy compound nucleus (sect. 2). The second treats the simultaneous break-up of the heated system in several IMFs (sect. 3). Depending on the excitation energy acquired in the first part of the reaction, the system either undergoes the simultaneous break-up or starts directly the sequential deexcitation cascade.

\section{Evaporation of IMFs}

In the de-excitation code ABLA, the compound nucleus at every step of its evolution has two possible decay channels: fission and evaporation. Up to now the evaporation part of ABLA considered only the emission of light particles. Here, we extended the evaporation to intermediate-mass fragments, i.e. to the emission of light nuclei with $Z>2$ up to the Businaro-Gallone maximum of the mass-asymmetry dependent potential. The statistical weight for the emission of these fragments is calculated on the basis of the detailed-balance principle. The decay width $(\Gamma)$ as a function of the excitation energy $(E)$ depends on the inverse cross section $\left(\sigma_{i n v}\right)$, on the level densities $\rho_{\text {imf }}$ and $\rho_{d}$ of the two final nuclei (the evaporated IMF and the remaining daughter nucleus) and on the level density of the compound nucleus above the ground state $\left(\rho_{C}\right)$ :

$$
\Gamma \approx \int_{0}^{E_{\text {inf }}^{\max }} \int_{0}^{E_{d}^{\max }} \sigma_{i n v} \frac{\rho_{\text {imf }}\left(E_{\text {imf }}\right) \cdot \rho_{d}\left(E_{d}\right)}{\rho_{C}(E)}(\varepsilon-B) d E_{i m f} d E_{d}
$$

with the following relation that guaranties the energy conservation:

$$
E=E_{\text {imf }}+E_{d}+Q+\varepsilon-B .
$$

Here $E, E_{\text {imf }}$ and $E_{d}$ represent the initial excitation energy of the mother nucleus, and the excitation energies of the IMF and daughter nucleus, respectively. $Q$ is the Q-value, $\varepsilon$ is the total kinetic energy in the centre of mass of the system, and $B$ is the barrier of the potential. The barrier $(B)$ was calculated using 
the empirical fusion nuclear potential of Bass [9]. The empirical nuclear potential contains some parameters, which were fixed by R. Bass. If the emitted particle is an IMF, the Bass results are well in agreement with the data. However, if the emitted particle is a proton or an alpha, the barrier seems to be overestimated. This problem was already discussed in the past and never solved [10-12]. In order to bypass the problem, in case of $\mathrm{p}$ and $\alpha$ emission, we used other parameters than those given by Bass. The parameters were determined by comparing the results with experimental proton and alpha absorption cross-sections on different nuclei. The thermal expansion of the compound nucleus [13] is considered. The inverse cross section $\left(\sigma_{i n v}\right)$ was calculated using the ingoing-wave boundary condition model [12], where only a real potential is used to describe the transmission probability of particles. An analytical approximation to equation (1) was used in order to avoid the numerical calculation of the two integrals, which is rather time-consuming. This technical procedure will be described elsewhere.

Since long time, it is discussed whether the emission of an IMF from a heavy nucleus (above the Businaro-Gallone point) is better described as an evaporation process or as a fission process with very asymmetric mass-split. Both approaches were already used in the past in nuclear deexcitation codes, e.g. in GEMINI [14] as very asymmetric fission or in GEM2 [15] as evaporation. Already in 1975 it was pointed out that there is a continuous transition between the two processes [16]. Recently [17] it was shown that even for such a heavy nucleus as ${ }^{238} \mathrm{U}$ the lightest IMFs are produced in a rather compact configuration, indicating that there is gradual transition from the standard fission process towards evaporation. In ABLA07 we based the fission-to-evaporation changeover on the M-shaped potential energy as a function of the mass asymmetry. At the point were the M-shaped potential reaches it maximum, the fission model smoothly fades away in favor of the evaporation process.

In figure 1, we present the comparison of our calculation with the experimental data, taken during the GSI campaign [18], for the reaction $1 \mathrm{GeV}$ protons on ${ }^{238} \mathrm{U}$. Since intranuclear-cascade codes are rather time-consuming for such a heavy nucleus, we used the nucleus-nucleus reaction code ABRABLA07, were the ABRA code, which treats the abrasion stage, is coupled with the deexcitation code ABLA07. Both codes were developed at GSI. The ABRA code includes the BURST [19] model, were the prefragments arising from high-energy nucleon-nucleus collisions are calculated. In the BURST model it is assumed that the impinging proton is digging a tunnel through the nucleus. As in the abrasion model, the characteristic of the compound nucleus depend on the number of removed nucleons. In this sense, the BRUST model can be considered as the abrasion process in case of spallation reactions. The result of the code is presented for the entire production range both on the chart of the nuclides (up) and as a charge distribution (down). The latter is compared with the experimental data. Please note that the measurement was technically limited to $Z \geq 7$, but the production of light nuclides extends even farther down to $Z=1$. The full line is obtained by the sum of the three components: the evaporated IMF, the fission fragments and the heavy evaporation residues (the evaporated light-charged particles $(Z \leq 2)$ are also
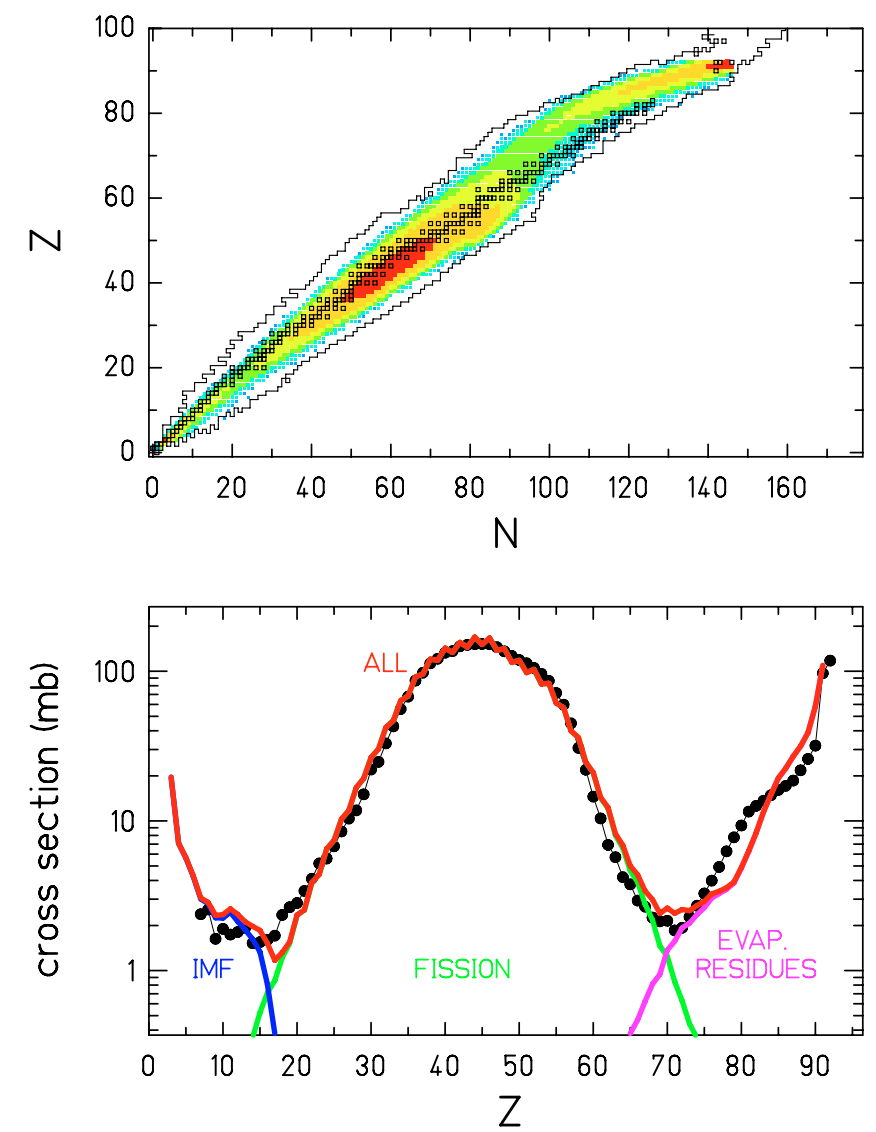

Fig. 1. Cross sections for the nuclei produced in $1 \mathrm{GeV}$ p on ${ }^{238} \mathrm{U}$. Up: Prediction of ABRABLA07 presented on the chart of the nuclides. Down: Experimental data (full dots) [1,17,20-22] are compared with the results of ABRABLA07 (solid line). The solid line is obtained by the sum of the three components: the evaporated IMF, the fission fragments and the heavy evaporation residues.

evaluated by the code, but not included in the figure here). The line corresponding to fission would represent the prediction with the previous version of ABLA. The progress is evident. In figure 2 , the mean $N / Z$-ratio and the standard deviations of the isotopic distributions are shown as a function of the atomic number for the entire production. The dashed line represents the stable isotopes, the solid line the result of the ABRABLA07 prediction. The overall agreement is very good.

\section{Break-up production of light fragments}

Although changes in the nuclear density of a compound nucleus normally are not considered in the modelling of spallation reactions, a nucleus tends to expand when it is heated. The most probable volume of a heated nucleus is given by the maximum of the number of states for a given excitation energy. The increase of volume changes the level density of the compound nucleus and affects the following deexcitation process. If the excitation energy acquired during the short collision stage is high enough, the increase of volume has more dramatic consequence: the nucleus enters the region of spinodal instabilities. That is to say that by lowering the 


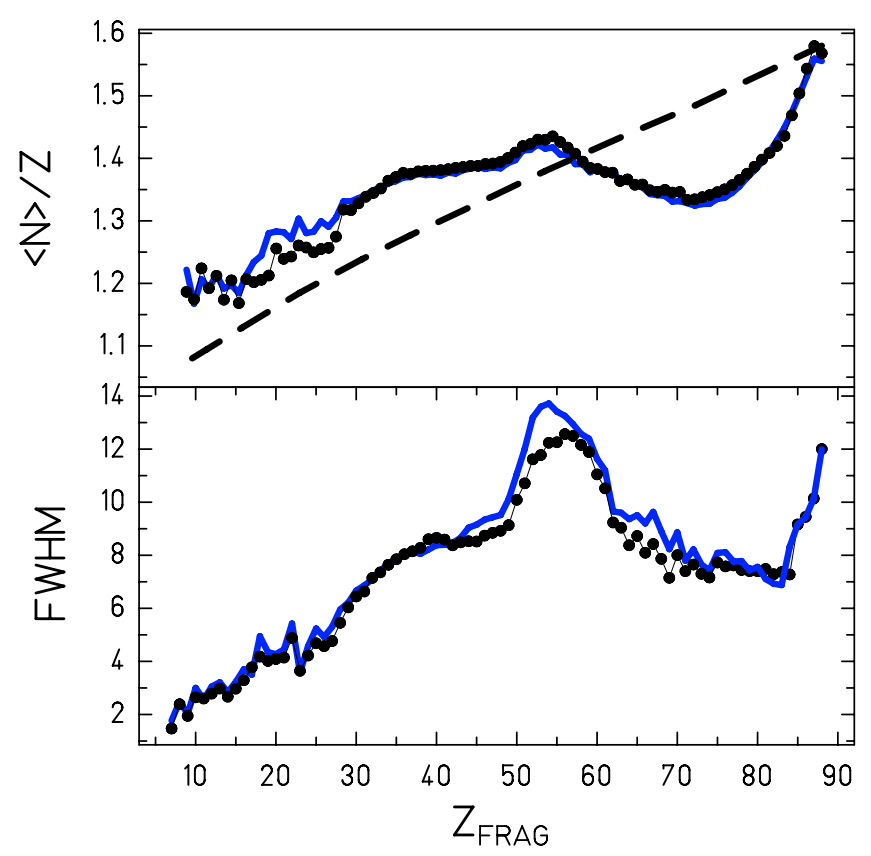

Fig. 2. Experimental mean $N / Z$ and FWHM of the isotopic distributions $(\bullet)$ for the reaction $1 \mathrm{GeV}$ p on ${ }^{238} \mathrm{U}$ as a function of the atomic number, compared with the ABRABLA07 prediction (solid line). The dashed line represents the stability line.

density, the nuclear matter divides into two phases: the liquid one, represented by a small amount of light nuclei (IMF) at normal nuclear density, and the gas phase, represented by individual nucleons. This process is often called "breakup". After break-up, the system explodes due to the repulsive Coulomb force among the fragments. Each fragment undergoes its deexcitation process and cools down. What is finally observed experimentally are the cold IMFs. The entire multifragmentation process is scientifically very interesting for its relation to the equation-of-state of nuclear matter, in particular to the liquid-gas phase transition.

It is not trivial to determine theoretically the size distribution of the break-up fragments. Models that evaluate it just by phase-space arguments, considering all possible partitions and weighted them by the number of available states, are considered to be inadequate since they neglect the dynamic of the expansion. On the other hand, the dynamics of the breakup process is far to be fully understood. In this context, in order to have a correct estimate of the production cross-sections of the IMFs, we based our model on the following considerations. Many experimental observations established that the production cross-sections in the domain of multifragmentation follow a power law:

$$
\frac{d \sigma}{d A_{I M F}} \propto A_{I M F}^{-\tau}
$$

Almost independently of the multiplicity, the mass distribution of intermediate-mass fragments is well described by a power law with an exponent $\tau \approx 2$. The value of $\tau$ turned to be rather universal. In the code ABLA07, it is assumed that the systems enters in the spinodal region when the excitation energy acquired during the first interaction stage (intra-nuclear cascade

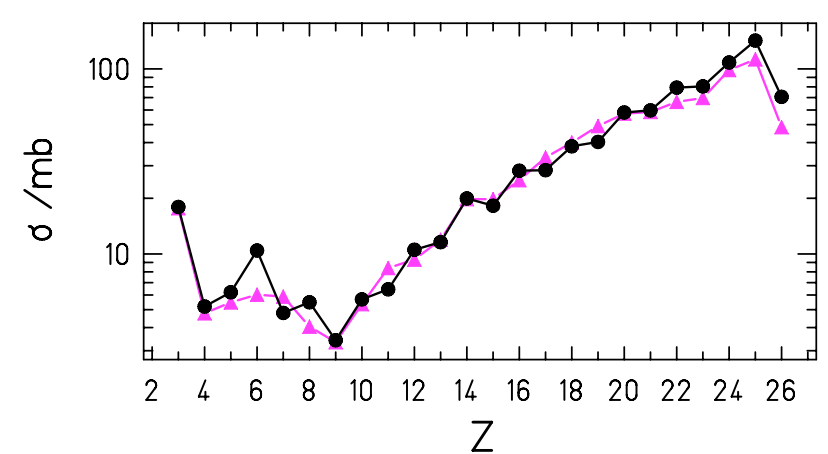

Fig. 3. Cross sections for the nuclei produced in the nuclear reaction $1 A \mathrm{GeV}$ protons on ${ }^{56} \mathrm{Fe}$. The experimental data $(\bullet)[1,20,21]$ are compared with the results of ABRABLA07 ( $\mathbf{\Lambda})$.

or abrasion) overcomes the value of about $3 \mathrm{MeV}$ per nucleon. As it was shown by the experiments who determined the caloric curve [23], when the nucleus exceeds the excitation energy of $3 \mathrm{MeV} / A$, the temperature of the system does not increase anymore, it remains approximately constant to the value of $5.5 \mathrm{MeV}$ (freeze-out temperature). The additional energy which is present in the nuclear system is used for the simultaneous emission of several intermediate-mass fragments and nucleons. The mass $A_{I M F}$ of any fragment produced at break-up is sampled from an exponential distribution with slope parameter $\tau \approx 2$, providing that the sampled mass is rejected when exceeding the maximum available mass. The sampling is performed several times until the entire mass is consumed. Each time, the charge $Z_{I M F}$ of the fragment is sampled from a Gaussian distribution centered at $Z_{\text {mean }}$, where $Z_{\text {mean }}$ is determined by imposing that the ratio $A / Z$ is the same of the hot remnant. The width of the distribution is given by the relation [24]:

$$
\sigma_{Z}^{2}=T_{\text {freeze-out }} / C_{\text {sym }}
$$

Where $C_{\text {sym }}$ is the symmetry term of the nuclear equation of state, set to $14.0 \mathrm{MeV}$ [25], and $T_{\text {freeze-out }}$ is the freeze-out temperature, set to $5.5 \mathrm{MeV}$ [26].

Please note that the procedure described above to determine the size distribution of the break-up IMFs has also the technical advantage of being very fast, an important issue for our original purpose since in nuclear technology small computation time is a value.

The case of $1 \mathrm{GeV}$ protons on ${ }^{56} \mathrm{Fe}[27,28]$ was studied. This a very interesting case, because both evaporation of light nuclei and break-up are responsible for the production of the IMFs, as it was found out by studying the kinematical properties of the final fragments $[13,29,30]$. Moreover, the study of this reaction is of interest in nuclear technology, since iron is an important construction material. In figures 3 and 4 , the charge distribution and nine isotopic distributions obtained experimentally are compared with the results of the ABRABLA07 code.

The charge distribution (fig. 3) shows that the overall prediction is satisfactorily, although structural effects connected to pairing are not well reproduced. Looking in detail to the isotopic distributions (fig. 4), it is interesting to note that both break-up and IMF evaporation contribute strongly to the 

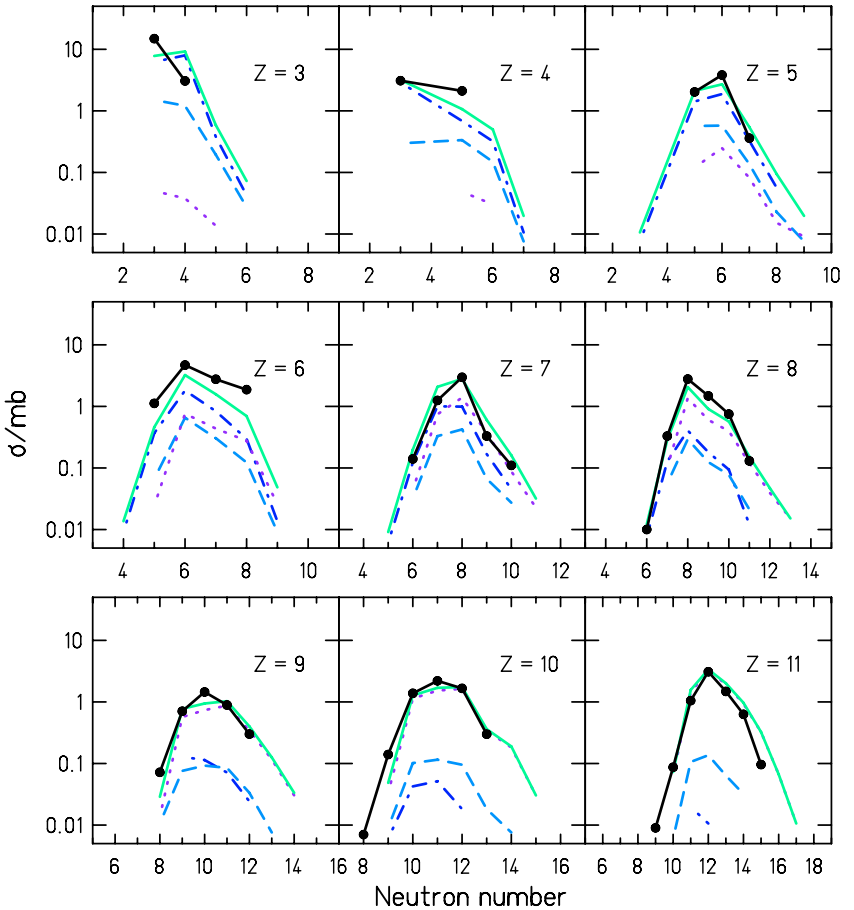

Fig. 4. Isotopic distributions for the lightest IMF of the reaction $1 A \mathrm{GeV}$ protons on ${ }^{56} \mathrm{Fe}$. The experimental data $(\bullet)[1,20,21]$ are compared with the results of ABRABLA07 (solid lines). The projectile residues, the break-up products, and the evaporated IMFs are represented by the dotted lines, by the dashed lines, and by the dashed-dotted lines, respectively.

production. The contribution of the break-up is maximum for the lightest nuclei and drops down rapidly as the size of the final fragment increases. Also the production by IMF evaporation is important for the lightest nuclei and vanishes away quickly in favour of the residual nuclei produced as end products of the evaporation cascade. In order to check whether the weight of the different mechanisms is correct one should compare the velocity spectra of the individual nuclides. The implementation of the kinematical properties of the fragments will be done in the near future.

\section{Conclusions}

The range of emitted fragments in the ABLA07 code was extended above $\mathrm{Z}=2$ in order to obtain a more realistic description of the production of intermediate-mass fragments
(IMFs), which was strongly underestimated in the previous version. Two mechanisms for the production of IMFs were implemented. In the first scenario, all nuclei below the BusinaroGallone maximum of the mass-asymmetry dependent barrier are taken into account in the evaporation process. In the second scenario, the simultaneous break-up of the system is modelled according to a power-law distribution. The modelling of both mechanisms is performed in such a way to keep the speedness of the code. The code was tested with a large amount of highprecision data measured at GSI. The results are good.

This work has been supported by the European Community with the contract FI6W-CT-2004-516520.

\section{References}

1. http://www.gsi.de/charms/data.htm.

2. http://www.fz-juelich.de/ikp/cosy/de.

3. http://www.fz-juelich.de/ikp/pisa.

4. Yu.E. Titarenko et al., Phys. Rev. C 65, 064610 (2002).

5. M. Gloris et al., Nucl. Instrum. Meth. 463, 593 (2001).

6. http://nuklear-server.fzk.de/eurotrans.

7. A. Kelic (these proceedings).

8. A. Boudard et al., Phys. Rev. C 66, 044615 (2002).

9. R. Bass, Nuclear Reactions with Heavy Ions (Springer, Berlin, 1980).

10. R.J. Charity et al., Phys. Rev. C 56, 873 (1997).

11. R.J. Charity et al., Phys. Rev. C 63, 024611 (2001).

12. M. Kildir et al., Phys. Rev. C 51, 1873 (1995).

13. K.-H. Schmidt, Bulg. J. Phys. 30 (2005).

14. R.J. Charity et al., Nucl. Phys. A 483, 371 (1988).

15. S. Furihata, Nucl. Instrum. Meth. B 171, 251 (2000).

16. L.G. Moretto, Nucl. Phys. A 247, 211 (1975).

17. M.V. Ricciardi et al., Phys. Rev. C 73, 014607 (2006).

18. K.-H. Schmidt et al. (these proceedings).

19. K.-H. Schmidt et al. (to be published).

20. J. Taieb et al., Nucl. Phys. A 724, 413 (2003).

21. M. Bernas et al., Nucl. Phys. A 725, 213 (2003).

22. M. Bernas et al., Nucl. Phys. A 765, 197 (2006).

23. J. Pochodzalla et al., Phys. Rev. Lett. 75, 1040 (1995).

24. A. Raduta, F. Gulminelli, Phys. Rev. C 75, 024605 (2007).

25. D. Henzlova et al., nucl-ex/0507003.

26. K.-H. Schmidt et al., Nucl. Phys. A 710, 157 (2002).

27. C. Villagrasa-Canton et al., Phys. Rev. C 75, 044603 (2007).

28. P. Napolitani, Ph.D. thesis, Université Paris XI, France, 2004.

29. P. Napolitani et al., Phys. Rev. C 70, 054607 (2004).

30. P. Napolitani, Bulg. J. Phys. 30, (2005). 